\title{
Special issue on information dissemination and new services in P2P systems
}

\author{
Min Song $\cdot$ Sachin Shetty $\cdot$ Wenbin Jiang $\cdot$ EK Park
}

Received: 5 October 2010 /Accepted: 11 October 2010 /Published online: 3 November 2010

(C) Springer Science + Business Media, LLC 2010

Information dissemination is an important $\mathrm{P} 2 \mathrm{P}$ application that has received considerable research attention in recent years. P2P information dissemination systems range from simple file sharing applications to more complex systems that allows users to securely and efficiently publish, organize, index, search, update and retrieve data in a distributed storage medium. For complex P2P information dissemination systems, there is a need for features which include security, anonymity, fairness, scalability, resource management, and organization capabilities. For effective information dissemination, following features of P2P systems and infrastructure need to be updated: distributed object location and routing mechanisms, novel approaches to content replication, caching and migration, encryption,

\author{
M. Song $(\bowtie)$ \\ Old Dominion University, \\ Norfolk, VA, USA \\ e-mail:msong@odu.edu \\ S. Shetty \\ Tennessee State University, \\ Nashville, TN, USA \\ e-mail: sshetty@tnstate.edu \\ W. Jiang \\ Huazhong University of Science and Technology, \\ Wuhan, Hubei, China \\ e-mail: jwbhust@gmail.com \\ E. Park \\ CUNY at College of Staten Island, \\ Staten Island, NY, USA \\ e-mail: ek.ek.park@gmail.com
}

authentication, access control, and resource trading and management schemes.

As P2P-based technologies play a more and more important role in the Internet, a number of new applications and services have been developed in the past few years, including file sharing, music downloading, live streaming, video on demand, and P2P based game platform. For these P2P systems, the key issues to be considered are scalability, robustness, reliability, and security. Moreover, today's networks are becoming increasingly more heterogeneous in terms of both network technologies and traffic, impacted by various factors including the wide deployment of wireless networks, diversification of multimedia formats, various requirements of different users, which brought many new challenges to P2P systems.

The Call for Papers attracted nine submissions worldwide. After a rigorous review process, five papers have been selected for publication. An outline of the selected papers is presented as follows.

The first article, by Jun-Hong Cui, et. al., attacks the problem of scalability, routing efficiency and complex query support for a P2P file sharing system. The authors propose a semantic overlay network of logical nodes, in which queries are routed on the basis of semantics. By exploiting the concepts of hierarchy and semantics, PSON has been demonstrated to support complex queries in a scalable and efficient way. The second article, by Zhipeng Ouyang, et. al., addresses a critical problem of factoring users' subjective preferences in large-scale video streaming applications. The authors derive a model and formulate the problem as a resource demand vs supply problem. A framework is presented to address the challenge via efficient bandwidth allocation and group cooperation. The 
third article, by Ansar-Ul-Haque Yasar, et. al., proposes a framework for context-aware adaptive information sharing that allows the evaluation of and comparison with alternative information routing protocols using network metrics to measure a variety of quality attributes of the information dissemination protocols. The framework has been evaluated with different information dissemination protocols in an inter-vehicle communication scenario. The fourth article, by Nikolaos Thomas Efthymiopoulos, et. al., proposes a dynamically reconfigurable overlay architecture that organizes its peers according to network locality information and heterogeneous uploading capabilities of them. The benefits of the optimized overlay are fully exploited by an efficient scheduler, which guarantees the complete and fast distribution of the stream and high uploading bandwidth utilization. The last article, by Linchen Yu, et. al., presents a new caching strategy that integrates memory-caching strategy with disk-caching schemes. By using the proposed strategy, peers can request media data from neighbors of the overlay, buffer the fresh part into the memory slots and the watched part into the free local disk, which can enlarge the capacity to buffer media data.

In closing, we would like to thank all anonymous reviewers who spent much of their precious time reviewing the papers. Their timely reviews and comments greatly helped us select the best papers for this special issue. We also thank all authors who have submitted their papers for consideration for this issue. Special thanks go to the Editor-in-Chief of Journal of Peer-to-Peer Networking and Applications, Professor Xuemin Shen, for offering us the opportunity to edit this special issue. For more information about this special issue, please visit http://PPNA.edmgr.com.

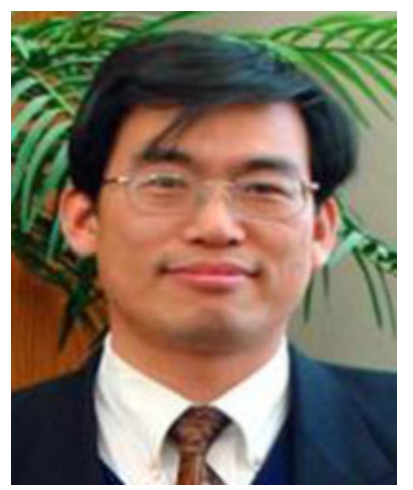

Min Song is an Associate Professor in the Department of Electrical and Computer Engineering at Old Dominion University. He received his $\mathrm{PhD}$ in Computer Science from the University of Toledo in 2001. His research interests include design, analysis, and evaluation of cognitive radio networks, wireless sensor networks, wireless mesh networks, WLAN, and mobile ad-hoc networks. Over the years, he has secured funding from NSF, DOE, NASA,

ODU, and local industry, and published numerous journal and conference papers. Dr. Song is the recipient of NSF CAREER Award and the recipient of DOE GAANN Award.

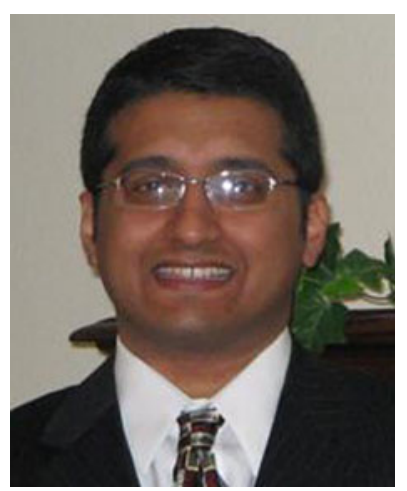

Sachin Shetty is an Assistant Professor in the Department of Electrical and Computer Engineering. He received his Ph.D. degree in Modeling and Simulation from Old Dominion University in 2007. His area of competency includes theoretical and experimental research in protocols design, performance analysis, security algorithms, virtual and augmented reality, and system implementation of wireless networks, cognitive networks, ad-hoc networks, and sensor networks. He has received external research funding exceeding \$1 million from the NSF, DoE, and the Tennessee Board of Regents for his research and educational innovations. He has authored and coauthored over 25 technical refereed and nonrefereed papers in various conferences, international journal articles, book chapters in research and pedagogical techniques.

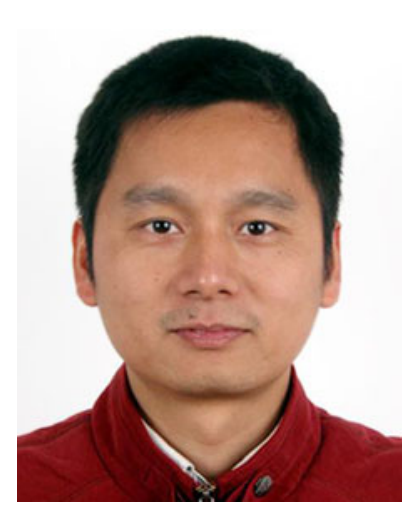

Wenbin Jiang is an Associate Professor at the School of Computer Science and Technology of the Huazhong University of Science and Technology (HUST) in China. Now he works in the Key Lab of Services Computing Technology and System Lab, Ministry of Edu, China. He received his $\mathrm{Ph}$. D. in information and communication engineering from HUST in 2004. His research interests focus on ubiquitous computing, data management, P2P computing, multimedia, etc. From 2005.12-

2006.1, he has been to Aizu University for VLIW research. He has been the PC chairs, Publicity chairs, publication chairs, PC members of more than 40 international conferences. He is a member of the Pervasive Computing Technical Committee of CCF, China, and a member of the IEEE Computer Society and a member of the IEEE Communication Society.

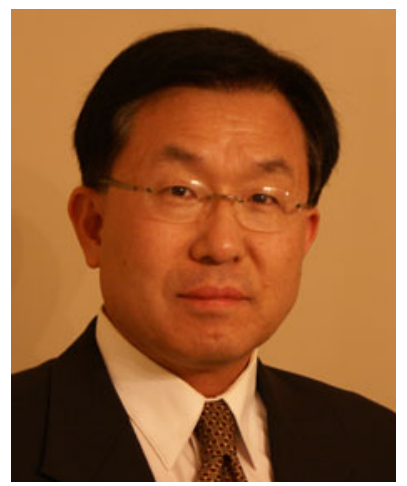

E. K. Park is a Professor of Computer Science at the City University of New York (CUNY) at $\mathrm{CSI}$. He received a $\mathrm{PhD}$ degree in Computer Science from the Northwestern University, Evanston, Illinois USA. His research interests include data mining, bioinformatics, information and knowledge management, computer communications and networks, optical networks, distributed systems, and object-oriented methodology. Currently, he is a Dean of Research and Graduate Studies at CSI/CUNY. He served as a Program Director, Division of Computing and Communications Foundations at US National Science Foundation. 